\title{
Lost to Follow-up and Predictors Among HIV-Exposed Infants in Northwest Ethiopia
}

\author{
Chalachew Adugna Wubneh (D) - Getaneh Mulualem Belay • \\ Fikadu Ambaw Yehualashet · Nigusie Birhan Tebeje • Birye Dessalegn Mekonnen • \\ Aklilu Endalamaw
}

Received: June 13, 2020 / Accepted: October 14, 2020 / Published online: October 28, 2020

(c) The Author(s) 2020

\begin{abstract}
Introduction: Even though advancement in mother-to-child HIV transmission prevention services is observed, many infants are lost to follow-up and could not access the full package of mother-to-child HIV transmission prevention services as a result. This is one of the obstacles to the effectiveness of the program. Therefore, determining the magnitude of lost to follow-up and its predictors is important among HIV-exposed infants.

Method: This institution-based retrospective cohort study was conducted from August 2013 to June 2018 at the University of Gondar Comprehensive Specialized Hospital. We
\end{abstract}

C. A. Wubneh $(\bowtie) \cdot$ G. M. Belay

Department of Pediatrics and Child Health Nursing, School of Nursing, College of Medicine and Health Sciences, University of Gondar, Gondar, Ethiopia e-mail: mekidem21@gmail.com

F. A. Yehualashet - N. B. Tebeje

Unit of Community Health Nursing, School of

Nursing, College of Medicine and Health Sciences,

University of Gondar, Gondar, Ethiopia

B. D. Mekonnen

Department of Nursing, Teda Health Sciences

College, Gondar, Ethiopia

A. Endalamaw

Department of Pediatrics and Child Health Nursing, School of Health Sciences, College of Medicine and Health Sciences, Bahir Dar University, Bahir Dar, Ethiopia retrieved charts of 423 child-mother pairs through a simple random sampling technique. Data collectors extracted data by using a data extraction tool adapted from the Ethiopian Federal Ministry of Health HIV-exposed infant follow-up form. Bivariable and multivariable Cox regression models were fitted to identify predictors of lost to follow-up.

Result: A total of 402 child-mother pairs were included in the study. Of the study participants, $6.0 \%$ were lost to follow-up for more than 3 months before the declaration of their HIV status. Born from rural residence mother $(\mathrm{AHR}=3.5$; 95\% CI 1.549-7.894), infants whose mothers have three and more children $(\mathrm{AHR}=$ 3; 95\% CI 1.284-6.963), and low birth weight infants (AHR $=3.2 ;$ 95\% CI 1.055-9.450) were independent predictors of lost to follow-up among HIV-exposed infants.

Conclusion: Significant numbers of infants were unable to access full HIV diagnosis and care services as a result of loss to follow-up. Special consideration for mothers having large numbers of children, rural residence, and low birth weight infants could be an important intervention to decrease lost to follow-up.

Keywords: Ethiopia; HIV-exposed infant; Lost to follow-up; Predictors 


\section{Key Summary Points}

\section{Why carry out this study?}

HIV-exposed infants are a vulnerable population for HIV infection and related complications as they need special care and support.

Lost to follow-up from service is one of the challenges to accessing the full package of mother-to-child HIV transmission prevention services for this vulnerable population.

This study tries to show the magnitude of lost to follow-up and contributing factors among HIV-exposed infants in relation to mother-to-child HIV transmission prevention services.

\section{What was learned from the study?}

A number of infants were unable to access full HIV diagnosis and care service as a result of lost to follow-up in health care services.

Infants born into a large family, from a rural residence, and with low birth weight were more prone to be lost from HIV care and support services.

Therefore, those infants who are at risk for lost to follow-up need special attention to retain them in the health care services.

\section{DIGITAL FEATURES}

This article is published with digital features, including a summary slide, to facilitate understanding of the article. To view digital features for this article go to https://doi.org/10.6084/ m9.figshare.13084586.

\section{INTRODUCTION}

It has been estimated that globally around 37.9 million people were living with human immunodeficiency virus (HIV) in 2018, and of these more than 23 million were receiving antiretroviral therapy (ART) [1]. In 2013, about 3.2 million children below the age of 15 years were living with HIV worldwide, $91 \%$ of them in sub-Saharan Africa [2]. In sub-Saharan Africa numerous HIV-infected infants and children died of HIV-related illnesses without knowing their HIV status. In the first one and a half years of life, nearly one-third of infants died because of poor access and utilization of ART, supportive care, and prophylactic drug treatments [3]. One of the prevention of mother-to-child transmission (PMTCT) interventions, early infant diagnosis (EID), has aimed to identify HIV-infected infants before the occurrence of clinical disease to enhance care and follow-up [4]. Lost to follow-up (LTFU) of HIV-infected and exposed individuals remains the major obstacle to the efficacious delivery of HIV care and support programs in sub-Saharan Africa [5].

In the absence of protective measures, the possibility of acquiring HIV during pregnancy and delivery is $15-30 \%$, and the risk will be raised to $20-45 \%$ with breastfeeding. The PMTCT schemes can minimize the vertical transmission of HIV to less than $1 \%$ in advanced nations; however, in spite of its positive progress, the success rate is poor in developing countries [6, 7]. Although the global rate of transmission of HIV reduced from $26 \%$ in 2009 to $10 \%$ in 2015, PMTCT remains a challenge for HIV control strategy [8].

In a public-funded urban hospital in Johannesburg, South Africa, almost half of the infants born to HIV-infected mothers in a routine PMTCT service were lost to follow-up by 2 weeks of age [9].

In spite of the development of knowledge of effective interventions to save the lives of HIVexposed infants, several exposed infants do not get the full package of amenities due to LTFU [10].

In Africa several studies have verified the huge number LTFU rate of children with an 
interval of $36.9 \%$ and $68.4 \%$; such unacceptably high LTFU prevalence experienced by PMTCT programs impedes the provision of appropriate interventions for HIV-infected children. This resulted in the poor success of PMTCT and is one of the main explanations for why more than $90 \%$ of the children who acquired HIV infection in 2011 live in sub-Saharan Africa [11].

In Malawi, the loss to follow-up rate of patients receiving HIV care and treatment has been unacceptably high. In 2012, about 100,624 patients $(18 \%)$ were lost to follow-up and therefore missed the opportunity of benefiting from ART care and support [12]. Other studies also reported that in Cameroon $47.37 \%$ and in Uganda $48 \%$ of infants were lost to follow-up $[13,14]$.

A study in Kenya reported that $22 \%$ of infants were LTFU at 18 months of age [15].

In Ethiopia, only $40 \%$ of HIV-positive pregnant women and less than a quarter of newborns of these women received ART prophylaxis. On the other hand about $11 \%$ of exposed infants received virologic investigation for HIV within 6 weeks of birth [16].

Several social, cultural, economic, and infrastructural factors contribute to the poor outcomes of PMTCT and a higher rate of LTFU. These factors are composite, with some involving the children themselves, the caregivers, the family structure and society, and the health care systems. There may not be single factors that appear to be replicated across the board because of differences in culture and approaches by various HIV programs across Africa. Some of the factors that have been linked to LTFU are discussed below. A study conducted in India showed that poor educational status, lower wealth quintile, and being registered after 20 weeks of gestation of pregnancy were predicting variables affecting LTFU [11]. A hospitalbased case-control study in Cameroon indicated that mothers who had no formal education were more likely to be lost to follow-up with HIV care programs compared to those who attended formal education [17].

Knowledge of the factors associated with LTFU has potential utility in deciding for patient retention in pediatric HIV care programs. Further, knowing the factors leading to
LTFU may provide unique opportunities for early intervention, to augment retention in HIV programs and improve overall outcomes of these children. But the magnitude of and factors associated with loss to follow-up of HIV-exposed infants are not yet investigated well in Ethiopia. Therefore, the main objective of this study was to determine the magnitude of and identify factors associated with loss to follow-up among HIV-exposed infants.

\section{METHODS}

\section{Study Setting}

The University of Gondar Comprehensive Specialized Hospital is expected to serve more than five million people from different parts of the country. Since 2005 the hospital has started providing HIV care and support including ART for children and adults, and PMTCT services for mothers and their children. After 2013 the hospital has started "option $\mathrm{B}+$ "- the new PMTCT approach for the mother and childcare as recommended by the Ethiopian Federal Ministry of Health. According to the Ethiopian Ministry of Health's national PMTCT guideline, all HIV-exposed infants must enroll in followup and care soon after delivery, and at 6 weeks PCR/DNA (polymerase chain reaction /deoxyribonucleic acid) analysis is conducted. ART initiation is determined on the basis of the result of the test [18]. If HIV negative, follow-up is continued up to 18 months until HIV negative is reconfirmed. Infants are expected to be discharged from the follow-up after the HIV rapid antibody test done at 18 months or after a 6-week cessation of breastfeeding.

\section{Study Design and Period}

A hospital-based retrospective cohort study was conducted from August 2013 to June 2018 in the University of Gondar Comprehensive Specialized Hospital, Northwest Ethiopia and the data were extracted from February to March 2019. 


\section{Study Population}

The study population comprised all mothers and children who were registered for HIV care between August 2013 and June 2018 at the University of Gondar Comprehensive Specialized Hospital, Northwest Ethiopia.

\section{Inclusion and Exclusion Criteria}

All children and mothers who have complete baseline registration were included in this study. (Baseline data comprised the sex, birth weight, and gestational age at birth of the infants and the age, ART starting time, baseline CD4 count, and baseline WHO clinical stage of the mothers.)

\section{Sampling Technique and Procedures}

The sample was determined by using a single population proportion formula and double proportion by using common factors from a previous study [9]. The largest sample size was 423 calculated from a single population proportion. The participants of this study were selected using simple random sampling technique. First, the total number of exposed children were identified from their registration number with the study. Then, study participants were selected using a computer-generated random number. After identifying the infant unique registration number, the mother unique registration numbers were accessed. Finally, all the necessary data variables were collected manually in the follow-up program.

\section{Operational Definitions}

HIV-exposed infant Infant born from HIVpositive mothers

Lost to follow-up Infants missing their appointment for more than 3 months

Censored Infants who transfer out, completed follow-up, attending follow-up according to schedule, and dead were considered as censored

\section{Data Collection Tools}

Data were extracted by using data the extraction tool customized from the Ethiopian Federal Ministry of Health's HIV-exposed infant followup chart. Data were collected from the charts and registration books of HIV-exposed infant-mother pairs.

\section{Data Quality Control Measures}

The data extraction tool was checked before the actual data collection period to ensure that data from the chart and follow-up registration book matched the information in the document and the data collection tool, and modification was made accordingly. Three PMTCT trained nurses were recruited for data collection. The data collection process was closely monitored by one MSc nurse supervisor on a daily basis to ensure the quality of data. One day of training was given to data collectors and the supervisor on objectives of the study, data collection tool, procedure, and ethical issues. Sociodemographic characteristics of mother-infant pairs, pregnancy, delivery, and clinical-related characteristics of mothers, and proportion of infants lost to follow-up were collected through the paper-based data collection tool.

\section{Data Processing and Analysis}

Data were collected first using the paper-based tool and then after collection the data were entered and coded using EPI Info version 7. Then, data were exported to SPSS version 20 for further analysis. The sociodemographic characteristics and proportion of lost to follow-up were described through descriptive statistics. Bivariable and multivariable Cox proportional hazard regression models were used to identify predictors of lost to follow-up. Variables with $p$ value $\leq 0.2$ in the bivariable analysis were fitted into the multivariable model. The Cox proportional hazard model assumption was checked by using the Schoenfeld residuals test. The adjusted hazard ratio (AHR) with a 95\% confidence interval (CI) was calculated. $p$ value 
$\leq 0.05$ was considered as statistically significant.

\section{Ethical Considerations}

This study was conducted in compliance with the 1964 Helsinki declaration and its later amendments or comparable ethical standards. The Institutional Review Board of Gondar approved this study. A permission and support letter was obtained from University of Gondar Comprehensive Specialized Hospital's chief clinical director after ethical approval and it was distributed to concerned offices. Since we used secondary data, it was impossible to get consent from mothers, and as result consent has been waived. Confidentiality of the information was maintained in hard and soft copy by locking and using a password as appropriate.

\section{RESULTS}

\section{Sociodemographic Characteristics of Mothers}

A total of 402 mother-child pairs were included in this study, which is $95 \%$ of the total sample size. Most of the mothers $(81.6 \%)$ were from urban areas and the majority (90\%) were Orthodox Christian religion followers. Nearly three-quarters $(76.9 \%)$ had attended at least primary education. Regarding the marital status of the mother, $72.1 \%$ were married. More than of half mothers $(61.7 \%)$ were between the ages of 19 and 30 years (Table 1 ).

\section{Sociodemographic and Health-Related Characteristics of HIV-Exposed Infants}

Of the $408 \mathrm{HIV}$-exposed infants included in the study, nearly half $(51.7 \%)$ were male. Almost all (99.5\%) of the HIV-exposed infants had good adherence to cotrimoxazole preventive therapy, which means the probability of missing a dose is 3 or less per month. Ninety-eight percent of the infants had taken daily prophylactic nevirapine for 6 weeks. Regarding birth weight, $66.2 \%$ had normal birth weight and $90.5 \%$ were term gestational age at birth. In the first
Table 1 Sociodemographic characteristics of mothers in University of Gondar comprehensive specialized hospital $(n=402)$

\begin{tabular}{lcc}
\hline Characteristics & Number & Percent (\%) \\
\hline Maternal age & 70 & 17.4 \\
$\quad<25$ years & 178 & 44.3 \\
25-30 years & 86 & 21.4 \\
30-35 years & 68 & 16.9 \\
$\quad \geq 35$ years & & \\
Marital status of mother & 290 & 72.1 \\
Married & 112 & 27.9 \\
Unmarried & & \\
Religion of mother & 362 & 90.0 \\
Orthodox Christian & 30 & 7.4 \\
Muslim & 10 & 2.6 \\
Protestant & & \\
Residence of mother & 328 & 81.6 \\
Urban & 74 & 18.4 \\
Rural & 134 \\
Occupation of mother & & \\
Government employed & 107 & 26.6 \\
Non-governmental employed & 133 & 33.1 \\
Housewife & 162 & 40.3 \\
Educational status of mother & & \\
No formal education & 93 & 23.1 \\
Primary education & 136 & 33.8 \\
Secondary education & 113 & 28.1 \\
College and above & 60 & 14.9 \\
Number of births & & \\
$\leq 2$ & & \\
$\geq 3$ & & \\
\hline
\end{tabular}

${ }^{a}$ Unmarried (single, widowed, divorced, separated)

6 months, most $(95.5 \%)$ of the infants were on exclusive breastfeeding and most of their parents $(92.5 \%)$ were alive. 
Table 2 Pregnancy, delivery, and clinical-related characteristics of mothers in University of Gondar comprehensive specialized hospital $(n=402)$

\begin{tabular}{|c|c|c|}
\hline Characteristics & Number & Percent (\%) \\
\hline \multicolumn{3}{|l|}{ ANC visit } \\
\hline No ANC & 23 & 5.7 \\
\hline First visit & 16 & 4.0 \\
\hline Second visit & 52 & 12.9 \\
\hline Third visit & 49 & 12.2 \\
\hline Fourth visit & 188 & 46.8 \\
\hline Fifth visit & 74 & 18.4 \\
\hline \multicolumn{3}{|l|}{ Place of delivery } \\
\hline Hospital & 358 & 89.1 \\
\hline Health center & 44 & 10.9 \\
\hline \multicolumn{3}{|l|}{ Mode of delivery } \\
\hline SVD & 347 & 86.3 \\
\hline CS & 55 & 13.7 \\
\hline \multicolumn{3}{|l|}{ ART starting time } \\
\hline Before pregnancy & 230 & 57.2 \\
\hline During pregnancy & 161 & 40.0 \\
\hline After labor & 11 & 2.7 \\
\hline \multicolumn{3}{|c|}{ Infant feeding practice in the first 6 months } \\
\hline $\mathrm{EBF}$ & 384 & 95.5 \\
\hline Non-EBF & 18 & 4.5 \\
\hline
\end{tabular}

$A N C$ antenatal care, $E B F$ exclusive breastfeeding, $S V D$ spontaneous vaginal delivery, $C S$ caesarean section, $A R T$ antiretroviral therapy

\section{Pregnancy, Delivery, and Clinical-Related Characteristics of Mothers}

From the reviewed maternal charts, the majority $(94.8 \%)$ of the mother had attended at least one antenatal care (ANC) and more than half $(57.2 \%)$ of the mothers had started ART before this pregnancy (Table 2 ).

\section{Magnitude of Lost to Follow-up Among HIV- Exposed Infants}

Out of 402, HIV-exposed infants, 24 (6.0\%) infants were lost to follow-up for more than
3 months before the final HIV status was determined. The infants were followed for a minimum of 3 months and a maximum 20 months with a median of 13 months of age. Nearly all (93.8\%) of the infants had enrolled in an HIV-exposed infant follow-up program within the first 6 weeks of life. Most (79.2\%) of the infants were lost to follow-up before their first birthday (Fig. 1).

\section{Predictors of Lost to Follow-up}

From the bivariable Cox regression analysis, residence of the mothers, number of births, educational status of the mothers, birth weight of the infant, and marital status of the mothers were associated with lost to follow-up among HIV-exposed infants. In the multivariable regression model, residence of the mothers, number of births, and birth weight of infants were independent predictors of lost to followup. Infants whose mothers live in rural areas were at 3.5 (95\% CI 1.549-7.894) times higher risk of lost to follow-up compared with infants whose mothers live in urban areas. Infants whose mothers have three or more children were at 3 (95\% CI 1.284-6.963) times higher risk for lost to follow-up. Infants whose birth weight was less than $2.5 \mathrm{~kg}$ were at 3.2 (95\% CI 1.055-9.450) times higher risk of lost to followup compared with infants whose weight is $2.5 \mathrm{~kg}$ or above (Table 3 ).

\section{DISCUSSION}

This study revealed that $6 \%(95 \%$ CI 3.7-8.2) of HIV-exposed infants were lost from HIV care follow-up. This implies that $6 \%$ of infants did not return for PMTCT services for at least 3 months before their final HIV status was declared. The finding is lower than studies conducted in Angola (19.3\%) [19], Cameroon (18\%) [20], Amhara region, Ethiopia (34\%) [21], Addis Ababa, Ethiopia (30.5\%) [22], Malawi (48\%) [23], Central Mozambique (50\%) [24], South region of Nigeria (67\%) [25], Uganda (43\%) [26], Brazil (15.4\%) [11], and North India (29\%) [27]. A possible reason for this discrepancy may be the difference in definition of lost 


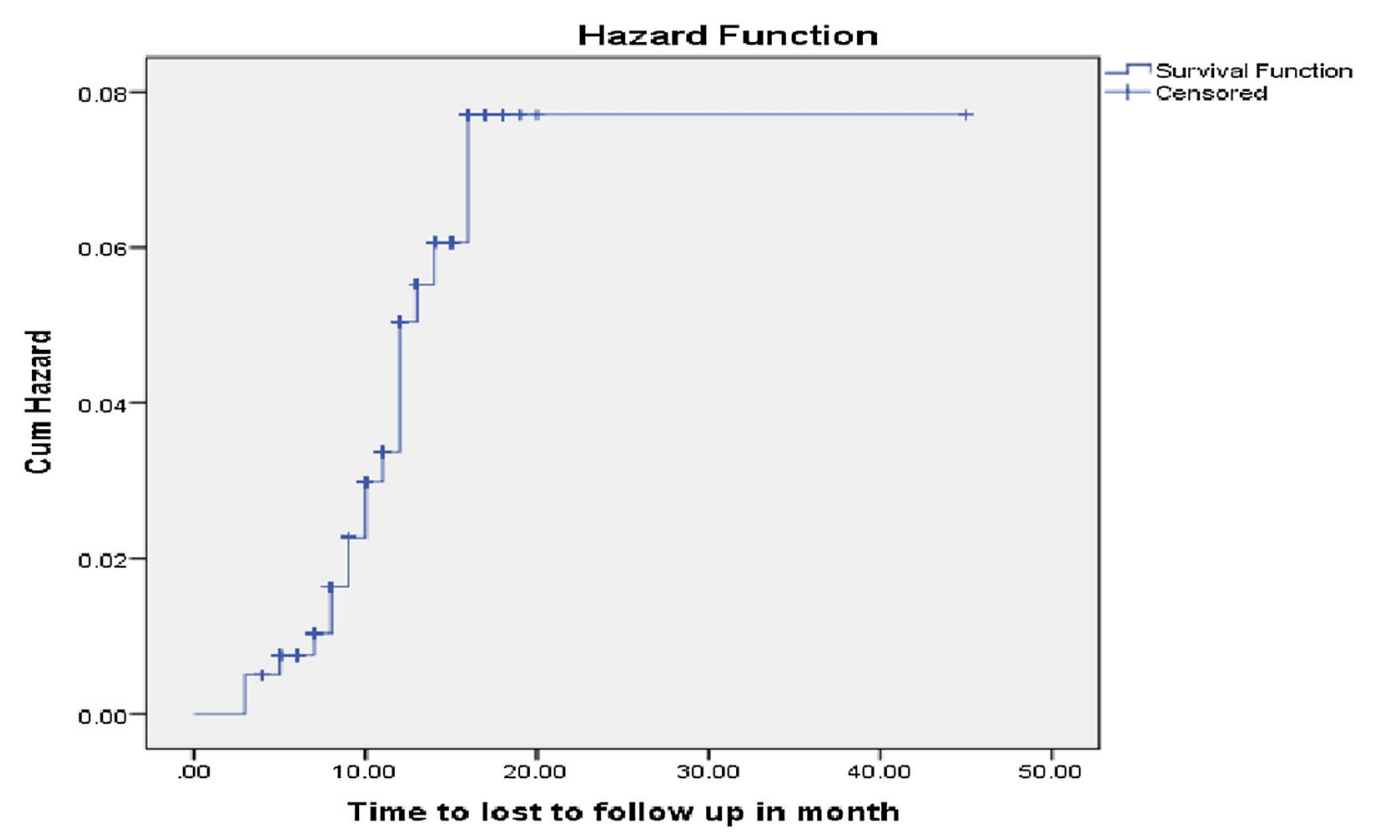

Fig. 1 Kaplan-Meier curve of proportion of lost to follow-up on the $y$-axis and time to lost to follow-up on the $x$-axis among HIV-exposed infants

to follow-up: in Brazil lost to follow-up was declared as absent from one schedule visit for PMTCT service and in Malawi missing from appointments for 2 months, but in this study lost to follow-up was defined as absent from PMTCT service for more than 3 months. Another possible explanation for this variation could be the change in intervention, as the aforementioned studies were conducted before the implementation of "option B+", which is a new PMTCT approach. This approach endorses initiating life-long ART for all pregnant, laboring, and lactating women, improving ART coverage, testing for HIV and starting highly active antiretroviral therapy (HAART) for all HIV-positive children, and early diagnosis for HIV-exposed infants. All this improves health service utilization that reduces the lost to follow-up of mothers and their child. This study also reported different predictors of lost to follow-up. Maternal residence, number of births, and birth weight were independent predictors of infants lost to follow-up. Infants whose mothers were from the rural residence were at 3.5 times higher risk of lost to follow-up as compared with infants whose mothers were from urban areas. This finding is supported by previous studies conducted in Brazil [11] and Zambezia, Mozambique [28]. Possible reasons for this difference may be due to distance from a health facility [29, 30], inaccessibility of transport [31], poor transport infrastructure [32], economical reasons for transport, and high working burden in the house for mothers [33-35]. Another issue that might affect this service utilization relates to fear of stigma and discrimination, which particularly affects the rural community [31]. Fear of stigma and discrimination could affect people in many ways; it may cause mothers to go far to find a health facility and the might decide to miss frequent visits to the health facility. On the other hand, fear of stigma and discrimination may end up with them not disclosing their status which directly hinders the PMTCT service utilization [36]. Another possible justification for the higher probability of lost to follow-up among rural residence infants might be related to poor access to other infrastructure, like electricity and telecommunication. In Ethiopia, accessibility to electricity and the network is poor, especially in rural areas. Poor electricity even for phone charging, not having a phone, and poor network access make it very challenging for early tracing systems for 
Table 3 Bivariable and multivariable Cox regression analysis of predictors of lost to follow-up among HIV-exposed infants in the University of Gondar Comprehensive Specialized Hospital, Northwest Ethiopia

\begin{tabular}{|c|c|c|c|c|c|}
\hline Characteristics & Category & $\begin{array}{l}\text { Lost to } \\
\text { follow-up }\end{array}$ & Censored & CHR (95\% CI) & AHR (95\% CI) \\
\hline \multirow[t]{2}{*}{ Residence of mother } & Urban & 13 & 315 & 1 & 1 \\
\hline & Rural & 11 & 63 & $\begin{array}{l}4.062 \\
\quad(1.650-9.997)\end{array}$ & $\begin{array}{l}3.497 \\
\quad(1.55-7.894)^{\mathrm{a}}\end{array}$ \\
\hline \multirow[t]{4}{*}{$\begin{array}{l}\text { Educational status of } \\
\text { mother }\end{array}$} & $\begin{array}{l}\text { No formal } \\
\text { education }\end{array}$ & 3 & 90 & 1 & 1 \\
\hline & Primary education & 7 & 129 & $\begin{array}{l}1.517 \\
\quad(0.392-5.867)\end{array}$ & $\begin{array}{l}1.737 \\
\quad(0.435-6.93)\end{array}$ \\
\hline & $\begin{array}{l}\text { Secondary } \\
\text { education }\end{array}$ & 5 & 108 & $\begin{array}{l}1.393 \\
\quad(0.333-5.831)\end{array}$ & $\begin{array}{l}2.32 \\
\quad(0.610-8.818)\end{array}$ \\
\hline & College and above & 9 & 51 & $\begin{array}{l}4.880 \\
\quad(1.32-18.037)\end{array}$ & $3.45(0.85-14.04)$ \\
\hline \multirow[t]{2}{*}{ Number of births } & $\leq 2$ & 11 & 247 & 1 & 1 \\
\hline & $\geq 3$ & 13 & 131 & $\begin{array}{l}2.023 \\
\quad(0.906-4.517)\end{array}$ & $2.989(1.28-6.96)^{\mathrm{a}}$ \\
\hline \multirow[t]{2}{*}{ Birth weight of infant } & $\geq 2.5 \mathrm{~kg}$ & 18 & 248 & 1 & 1 \\
\hline & $<2.5 \mathrm{~kg}$ & 6 & 130 & $\begin{array}{l}1.467 \\
\quad(0.582-3.699)\end{array}$ & $\begin{array}{l}3.157 \\
\quad(1.055-9.45)^{\mathrm{a}}\end{array}$ \\
\hline \multirow[t]{2}{*}{ Marital status of mother } & Married & 20 & 270 & 1 & $1^{\mathrm{a}}$ \\
\hline & Unmarried & 4 & 108 & $\begin{array}{l}1.962 \\
\quad(0.671-5.742)\end{array}$ & $\begin{array}{l}1.399 \\
\quad(0.517-3.784)\end{array}$ \\
\hline
\end{tabular}

${ }^{a}$ The highlighted AHR values are statistically significant

infants lost from their HIV care follow-up [37]. Another maternal-related factor that predicts infants lost to follow-up was having large numbers of children. Those infants from mothers having three and more children were at three times higher risk for lost to follow-up compared with mothers having fewer than three children. This may be because those mothers having large numbers of children may not have adequate time to take their exposed infants to every scheduled follow-up for a longer duration of time. Another possible reason may be that mothers having large numbers of children may be the only care provider for the remaining children in the home; as a result, it may be challenging for her to take the exposed infant to the health facility [30]. The third predictor for infants with lost to follow-up was being of low birth weight. Those infants weighing less than $2.5 \mathrm{~kg}$ were at 3.2 times higher risk for lost to follow-up than infants weighing $2.5 \mathrm{~kg}$ and above. A possible reason for this may be that low birth weight infants may have more frequent illness than those with normal birth weight. This may force mothers to seek recurrent health facility visits in addition to the scheduled follow-up, which creates a double burden for the mothers. Another possible reason may be that those low birth weight infants may suffer from illness and as a result 
the mother may visit different religious, cultural, and traditional healers, and this may detach the mother from health care visits. As a result, the infants may be absent from scheduled visits for PMTCT [38, 39].

This study has tried to include data for mother-infant pairs from the hospital starting from the implementation of the new PMTCT approach, which is "option $\mathrm{B}+$ ". Therefore, this study suggests that the implementation of "option $\mathrm{B}+$ " has shown a promising effect in the reduction of lost to follow-up among HIVexposed infants. This study has limitations: it involves data from a single hospital and is not multicenter; other limitations are that it could not include the reason for lost to follow-up and tracing was not conducted by investigators.

\section{CONCLUSION}

Significant numbers HIV-exposed infants did not attend the full PMTCT package because of loss to follow-up. Special attention should be given for those mothers having large numbers of children and from rural residence; and prevention of occurrence of low birth weight and further treatment early whenever it occurs. Improvement of the tracing system for those mothers from rural and remote areas, mothers having large numbers of children, and low birth weight infants could be a strategy for reduction of loss to follow-up for HIV-exposed infants.

\section{ACKNOWLEDGEMENTS}

The authors acknowledge data collectors, supervisors and University of Gondar Comprehensive Specialized Hospital.

Funding. Financial support was obtained from University of Gondar for the purpose of data collection. No funding or sponsorship was received for the publication of this article.

Authorship. All named authors meet the International Committee of Medical Journal Editors (ICMJE) criteria for authorship for this article, take responsibility for the integrity of the work as a whole, and have given their approval for this version to be published.

Disclosures. Chalachew Adugna Wubneh, Getaneh Mulualem Belay, Fikadu Ambaw Yehualashet, Nigusie Birhan Tebeje, Birye Dessalegn Mekonnen and Aklilu Endalamaw declare that they have no conflict of interest.

Compliance with Ethics Guidelines. This study was conducted in compliance with the 1964 Helsinki declaration and its later amendments or comparable ethical standards. The Institutional Review Board of Gondar approved this study. A permission and support letter was obtained from University of Gondar Comprehensive Specialized Hospital's chief clinical director after ethical approval and it was distributed to concerned offices. Since we used secondary data, it was impossible to get consent from mothers, and as result consent has been waived. Confidentiality of the information was maintained in hard and soft copy by locking and using a password as appropriate.

Data Availability. The datasets generated during and/or analyzed during the current study are available from the corresponding author on reasonable request.

Open Access. This article is licensed under a Creative Commons Attribution-NonCommercial 4.0 International License, which permits any non-commercial use, sharing, adaptation, distribution and reproduction in any medium or format, as long as you give appropriate credit to the original author(s) and the source, provide a link to the Creative Commons licence, and indicate if changes were made. The images or other third party material in this article are included in the article's Creative Commons licence, unless indicated otherwise in a credit line to the material. If material is not included in the article's Creative Commons licence and your intended use is not permitted by statutory regulation or exceeds the permitted use, you will need to obtain permission directly from the copyright holder. To view a copy of this licence, visit http://creativecommons.org/licenses/bync/4.0/. 


\section{REFERENCES}

1. UNAIDS, Global, HIV. AIDS statistics-2018 fact sheet. 2019. website. https://www.unaids.org/en/ resources/fact-sheet.

2. UNAIDS. 2013 progress report on the global plan towards the elimination of new HIV infections among children by 2015 and keeping their mothers alive. Geneva: UNAIDS; 2013.

3. Ciaranello AL, Park J-E, Ramirez-Avila L, Freedberg KA, Walensky RP, Leroy V. Early infant HIV-1 diagnosis programs in resource-limited settings: opportunities for improved outcomes and more cost-effective interventions. BMC Med. 2011;9(1): 59.

4. Hassan AS, Sakwa EM, Nabwera HM, et al. Dynamics and constraints of early infant diagnosis of HIV infection in rural Kenya. AIDS Behav. 2012;16(1):5-12.

5. Braitstein P, Katshcke A, Shen C, et al. Retention of HIV-infected and HIV-exposed children in a comprehensive HIV clinical care programme in Western Kenya. Trop Med Int Health. 2010;15(7):833-41.

6. De Cock KM, Fowler MG, Mercier E, et al. Prevention of mother-to-child HIV transmission in resource-poor countries: translating research into policy and practice. JAMA. 2000;283(9):1175-82.

7. Update WP. Use of antiretroviral drugs for treating pregnant women and preventing HIV infection in infants. Geneva: WHO; 2012.

8. Kyaw KWY, Oo MM, Kya NTT, et al. Low mother-tochild HIV transmission rate but high loss-to-followup among mothers and babies in Mandalay, Myanmar; a cohort study. PLoS One. 2017;12(9): e0184426.

9. Chetty TKS, Giddy J, Crankshaw TL, Butler LM, Newell M-L. A retrospective study of Human Immunodeficiency Virus transmission, mortality and loss to follow-up among infants in the first 18 months of life in a prevention of mother-to-child transmission programme in an urban hospital in KwaZulu-Natal, South Africa. BMC Pediatrics. 2012;12(1):146.

10. Sibanda EL, Weller IVD, Hakim JG, Cowan FM. The magnitude of loss to follow-up of HIV-exposed infants along the prevention of mother-to-child HIV transmission continuum of care: a systematic review and meta-analysis. AIDS. 2013;27(17): 2787-97.

11. da Cruz Gouveia PA, da Silva GAP. Predictors of loss to follow-up among children registered in an HIV prevention mother-to-child transmission cohort study in Pernambuco, Brazil. BMC Public Health. 2014;14(1):1232.

12. World Health Organization. Facilitating follow-up and retention among HIV+ mothers and exposed infants in Malawi-CHAI's mother-infant pair clinic. Geneva: WHO; 2013.

13. Efeutmecheh RS, Tiotsia AT, Djeunang GD, et al. The problem of lost to follow-up of mother-child pairs enrolled in the PMTCT program in Dschang District Hospital-Cameroon. Ig Sanita Pubbl. 2018;74(4):337-47.

14. Ankunda R, Cumber SN, Atuhaire C, et al. Loss to follow-up and associated maternal factors among HIV-exposed infants at the Mbarara Regional Referral Hospital, Uganda: a retrospective study. BMC Infect Dis. 2020;20(1):1-9.

15. Goggin K, Hurley EA, Staggs VS, et al. Rates and predictors of HIV-exposed infants lost to follow-up during early infant diagnosis services in Kenya. AIDS Patient Care STDs. 2019;33(8):346-53.

16. Wudineh F, Damtew B. Mother-to-child transmission of HIV infection and its determinants among exposed infants on care and follow-up in Dire Dawa City, Eastern Ethiopia. AIDS Res Treat. 2016;2016: 3262746.

17. Kigen HT, Galgalo T, Githuku J, et al. Predictors of loss to follow up among HIV-exposed children within the prevention of mother to child transmission cascade, Kericho County, Kenya. Pan Afr Med J. 2018;30:178.

18. Ethiopian Federal Ministry of Health. Comprehensive Integrated National PMTCT/MNCH/SRH, Guideline. Addis Ababa: FMOH; 2014.

19. Lussiana C, Clemente SVL, Ghelardi A, Lonardi M, Tarquino IAP, Floridia M. Effectiveness of a prevention of mother-to-child HIV transmission programme in an urban hospital in Angola. PLoS One. 2012;7(4):e36381.

20. Njom Nlend AE, Same Ekobo C, Bitoungui M, et al. Early outcomes of HIV exposed children in the first district-wide programme using extended regimens for the prevention of mother-to-child transmission of HIV, in Yaounde, Cameroon. J Trop Pediatrics. 2011;58(4):297-302.

21. Kebede B, Gebeyehu A, Jain S, Sun S, Haubrich R. Delay in early infant diagnosis and high loss to follow-up among infant born to HIV-infected women in Ethiopia. World J AIDS. 2014;4(04):402.

22. Shargie MB, Eek F, Abaychew A. Prophylactic treatment uptake and compliance with 
recommended follow up among HIV exposed infants: a retrospective study in Addis Ababa, Ethiopia. BMC Res Notes. 2011;4(1):563.

23. Ng'ambi WF, Ade S, Harries AD, et al. Follow-up and programmatic outcomes of HIV-exposed infants registered in a large HIV centre in Lilongwe, Malawi: 2012-2014. Trop Med Int Health. 2016;21(8):995-1002.

24. Napúa M, Manuel J, Vieira LC, et al. Reducing loss to follow-up of HIV exposed infants in Central Mozambique. Ann Global Health. 2016;82(3): 377-8.

25. Anoje C, Aiyenigba B, Suzuki C, et al. Reducing mother-to-child transmission of HIV: findings from an early infant diagnosis program in south-south region of Nigeria. BMC Public Health. 2012;12(1): 184.

26. Kiyaga C, Narayan V, McConnell I, et al. Retention outcomes and drivers of loss among HIV-exposed and infected infants in Uganda: a retrospective cohort study. BMC Infect Dis. 2018;18(1):416.

27. Seth A, Chandra J, Gupta R, Kumar P, Aggarwal V, Dutta A. Outcome of HIV exposed infants: experience of a regional pediatric center for HIV in north India. Indian J Pediatrics. 2012;79(2):188-93.

28. Cook RE, Ciampa PJ, Sidat M, et al. Predictors of successful early infant diagnosis of HIV in a rural district hospital in Zambezia, Mozambique. J Acquir Immune Defic Syndr. 2011;56(4):e104.

29. Timerga G, Hailemeskel S. Trend analysis on maternal health care services utilization in Amhara Region Referral Hospitals, Ethiopia. Int J Nutr Food Sci. 2018;7(3):81.

30. Awoyemi T, Obayelu O, Opaluwa H. Effect of distance on utilization of health care services in rural Kogi State, Nigeria. J Human Ecol. 2011;35(1):1-9.

31. Akal CG, Afework DT. Status of prevention of mother-to-child transmission (PMTCT) services utilization and factors affecting PMTCT service uptake by pregnant women attending antenatal care clinic in selected health facilities of Afar Regional State, Ethiopia. J Environ Public Health. 2018;2018:5127090.

32. Sisay A. Infrastructure provision as a tool in ruralurban linkages: a case of Modjo and Lume area. Unpublished master's thesis, Urban Management, Ethiopian Civil Service University, Addis Ababa, Ethiopia; 2009.

33. Tarekegn SM, Lieberman LS, Giedraitis V. Determinants of maternal health service utilization in Ethiopia: analysis of the 2011 Ethiopian Demographic and Health Survey. BMC Pregnancy Childbirth. 2014;14(1):161.

34. Gebre E, Worku A, Bukola F. Inequities in maternal health services utilization in Ethiopia 2000-2016: magnitude, trends, and determinants. Reprod Health. 2018;15(1):119.

35. Tiruneh FN, Chuang K-Y, Chuang Y-C. Women's autonomy and maternal healthcare service utilization in Ethiopia. BMC Health Serv Res. 2017;17(1): 718.

36. Turan JM, Nyblade L, Monfiston P. Stigma and discrimination as barriers to PMTCT and HIV care and treatment for maternal, neonatal and child health. AIDS. 2012:22-7.

37. Atnafu A, Otto $\mathrm{K}$, Herbst $\mathrm{CH}$. The role of mHealth intervention on maternal and child health service delivery: findings from a randomized controlled field trial in rural Ethiopia. Mhealth. 2017;3:39.

38. Mekonnen Y, Mekonnen A. Utilization of maternal health care services in Ethiopia. 2002. Calverton: ORC Macro.

39. Goldman N, Heuveline P. Health-seeking behaviour for child illness in Guatemala. Trop Med Int Health. 2000;5(2):145-55. 